\title{
REPLY
}

\section{Gout treatment-more tablets might be needed}

\section{Fernando Perez-Ruiz and Ana Maria Herrero-Beites}

We thank Dr. Alvarez-Lario and Dr Alonso-Valdivieso for their kind comments about our paper (Crystal arthritis: Environment and genetics in gout: a maze for clinicians? Nat. Rev. Rheumatol. 10, 8-9; 2014), ${ }^{1}$ which raises some important issues (Gout treatment: when a tablet is not enough. Nat. Rev. Rheumatol. doi: $10.1038 /$ nrrheum.2013.173-c1). ${ }^{2}$ We did not extensively discuss the impact of lifestyle changes because it was not the topic of the paper. Our critical approach to that topic is available elsewhere. ${ }^{3}$ Nevertheless, we would like to make some points clear.

To sustain their argument, the authors reference an open, uncontrolled study, including a total of 13 patients (16-week duration) who underwent dietary intervention. ${ }^{4}$ The results have to be cautiously analysed-the patients had an extraordinarily high rate of flares in the 4 months before entering the study (2.1 per month); despite the mean serum urate at the end of the study $(470 \mu \mathrm{mol} / 1[7.8 \mathrm{mg} / \mathrm{dl}])$, the rate of flares dramatically sunk to 0.6 per month (a 71\% decrease), a reduction that even surpasses canakinumab in clinical trials. ${ }^{5}$ Finally, the short-term weight-loss observed is not necessarily associated with maintenance of such loss in the longterm, as shown by the cited paper. ${ }^{6}$ In the Multiple Risk Factor Intervention Trial, at 7 -year follow-up, only $14.39 \%$ of patients had lost $\geq 5 \mathrm{~kg}$, and $73 \%$ had a BMI of $>25$ at baseline. ${ }^{6}$ In a large, follow-up of a cohort of patients with gout, only $13 \%$ of patients withdrew from urate-lowering medications in the long-term and maintained normal serum urate levels; weight loss, but also other environmental factors, was associated with this outcome. ${ }^{7}$

The authors remark that the benefit of proper control of comorbid conditions might be of more benefit than the mere control of urate, referencing ACR recommendations. ${ }^{8}$ We assume they regard comorbid conditions as cardiovascular risk factors. Quoting the 2012 ACR recommendations, the panel "...recognized that diet and lifestyle measures alone provide therapeutically insufficient serum uratelowering effects or gout attack prophylaxis for a large fraction of individuals with gout." ${ }^{\prime \prime}$ In addition, we completely agree that proper control of comorbid conditions, such as diabetes, hypertension and hyperlipidaemia, should be addressed; in a considerable number of cases they will not be properly controlled with lifestyle modifications alone, and therefore a tablet for hyperuricaemia will not be enough-more tablets might be needed.

Alvarez-Lario and Alonso-Valdivieso support their assertions by referencing two educational interventions, including lifestyle education. ${ }^{9,10}$ Specific results of lifestyle intervention were not reported in either of these papers and $80-100 \%$ of patients were prescribed increasing doses of urate-lowering medications, and switched to other drugs if needed to achieve proper urate control. In these studies, education centred mostly on knowledge of the disease, therapeutic targets, outcomes ("curing gout"), and correct implementation of prescriptions. In one particular gout clinic, similar figures were reported, with almost $80 \%$ of patients achieving proper control of urate at a 4 -year follow-up. ${ }^{11}$ It is of great interest that such interventions are far better than results from audits of the management of gout. ${ }^{12}$

We agree that environmental modification interventions in gout might be critical, and we consider the education of doctors (a powerful environmental factor), in addition to that of patients, as especially important. ${ }^{13}$

Servicio de Reumatología and BioCruces Health Research Institute, Cruces University Hospital, Plaza Cruces S/N, 48903 Barakaldo, Spain (F. Perez-Ruiz). Physical Medicine Division, Hospital de Górliz, Astondo Ibitoki 2, Górliz, Spain (A. M. Herrero-Beites). Correspondence to: F. Perez-Ruiz fperezruiz@telefonica.net

\section{Acknowledgements}

This paper has been partially granted $(9 / 13)$ by Asociación de Reumatologos del Hospital de Cruces.
Competing interests

F. Perez-Ruiz declares that he has acted as a consultant for AstraZeneca, Menarini, Novartis, Metabolex, Pfizer, and SOBI. A. M. Herrero-Beites declares no competing interests.

1. Perez-Ruiz, F. \& Herrero-Beites, A. M. Crystal arthritis: Environment and genetics in gout: a maze for clinicians? Nat. Rev. Rheumatol. 10, 8-9 (2014).

2. Álvarez-Lario, B. \& Alonso-Valdivielso, J. L. Gout treatment: when a tablet is not enough. Nat. Rev. Rheumatol. http://dx.doi.org/10.1038/ nrrheum.2013.173-c1.

3. Perez Ruiz, F \& Herrero-Beites, A. M. Evaluation and treatment of gout as a chronic disease. Adv. Ther. 29, 935-946 (2012).

4. Dessein, S. H., Shipton, E. A., Stanwix, A. E., Joffe, B. I. \& Ramokgadi, J. Beneficial effect of weight loss associated with moderate calorie/ carbohydrate restriction and increased proportional of protein and unsaturated fat on serum urate and lipoprotein levels in gout. Ann. Rheum. Dis. 59, 539-543 (2000).

5. Perez-Ruiz, F., Chinchilla, S. P. \& HerreroBeites, A. M. Canakinumab for gout: a specific, patient-profiled indication. Expert Rev. Clin. Immunol. (in press).

6. Zhu, Y., Zhang, Y. \& Choi, H. K. The serum urate lowering impact of weight loss among men with a high cardiovascular risk profile: the Multiple Risk Factor Intervention Trial. Rheumatology (Oxford) 49, 2391-2399 (2010).

7. Perez-Ruiz, F., Herrero-Beites, A. M. \& Carmona, L. A two-stage approach to the treatment of hyperuricemia in gout: The "Dirty Dish" hypothesis. Arthritis Rheum. 63, 4002-4006 (2011).

8. Khanna, D. et al. 2012 American College of Rheumatology guidelines for management of gout. Part 1: Systematic nonpharmacologic and pharmacologic therapeutic approaches to hyperuricemia. Arthritis Care Res. (Hoboken) 64, 1431-1446 (2012).

9. Lim, A. et al. Achieving treat to target in gout: a clinical practice improvement project. Scand. J. Rheumatol. (2012).

10. Rees, F., Jenkins, W. \& Doherty, M. Patients with gout adhere to curative treatment if informed appropriately: proof-of-concept observational study. Ann. Rheum. Dis. 72 , 826-830 (2013).

11. Perez-Ruiz, F. et al. Tophaceous gout and high level of hyperuricaemia are both associated with increased risk of mortality in patients with gout. Ann. Rheum. Dis. 73, 177-182 (2014).

12. Perez-Ruiz, F. et al. An audit of the variability of diagnosis and management of gout in the rheumatology setting: The Gout Evaluation and Management Study. J. Clin. Rheumatol. 17, 349-355 (2011).

13. Doherty, M. et al. Gout: why is this curable disease so seldom cured? Ann. Rheum. Dis. 71, 1765-1770 (2012). 